\title{
STATUS OF THE HIGH ENERGY SC LINAC FOR THE TRASCO PROGRAM
}

\author{
C. Pagani, D. Barni, G. Bellomo, A. Bosotti, P. Michelato, R. Paulon, \\ P. Pierini, D. Sertore, G. Varisco, INFN Milano - LASA, Italy
}

\begin{abstract}
The status of the activities for the superconducting high energy linac of the TRASCO Program for an ADS system for nuclear waste transmutation is presented here. The design of a $1-2 \mathrm{GeV}, 30 \mathrm{~mA}$, linac at $704.4 \mathrm{MHz}$ is briefly discussed. The results on the cavity prototypes of the low$\beta$ cavities $(\beta=0.5)$ are reported.
\end{abstract}

\section{THE TRASCO PROGRAM}

TRASCO [1] is a program funded by the Italian Ministry of Research and University for the feasibility study and component prototyping of an Accelerator Driven System (ADS) for nuclear waste transmutation. INFN (for the accelerator) and ENEA (for the sub-critical reactor assembly) jointly manage the program, with the participation of many Italian companies and Universities.

The reference accelerator solution chosen for the TRASCO studies is a $30 \mathrm{~mA} 1 \mathrm{GeV}$ linac, composed of a proton source, an RFQ, and a superconducting (SC) linac.

\section{R\&D ACTIVITIES ON THE HIGH ENERGY SC LINAC}

Starting from beam energies in the range of 85$100 \mathrm{MeV}$ the use of SC multi-cell elliptical cavities appears the most efficient and cost-effective solution. The high-energy part of the TRASCO linac is composed of three sections, covering each an energy range with a single type of structure. The $\beta$ values of these structures are $0.5,0.65$ and 0.86 , respectively.

\subsection{General linac layout}

The transition energies between the sections are set to approximately 200 and $500 \mathrm{MeV}$, and the last family of cavities can operate up to approximately $2 \mathrm{GeV}$ without great losses of efficiency. The transverse focussing lattice is provided by a periodic array of quadrupole doublets, and the SC cavity cryostats are placed in the long drifts between quads. A conceptual design of these cryostats (based on TESLA) is one of the TRASCO goals.

\subsection{The cavity design}

The SC cavity design [2] was performed in collaboration with the French ASH group [3], under a MOU agreement that allows sharing the R\&D and prototyping efforts. In particular, while most of the effort of the French group is concentrated on the work on $\beta=0.65$ cavity prototypes [4], our group works on the development of $\beta=0.5$ cavities.
The baseline cavity design geometries have been chosen using a procedure that takes into account all the electromagnetic and mechanical [5] performances of the cavities, and choosing the most suitable compromise for the required application.

\subsection{Cavity prototypes activities for TRASCO}

The TRASCO cavity prototypes are being built by Zanon [6], which is an Industrial partner of the TRASCO program. Up to now, two single-cell $\beta=0.5$ cavities with reactor grade (low RRR) niobium have been built mainly for the testing of all the fabrication tooling.

Of these cavities, the first one has already been tested in a vertical cryostat and its performances are discussed in the following paragraphs. Figure 1 shows the first two TRASCO cavity prototypes.
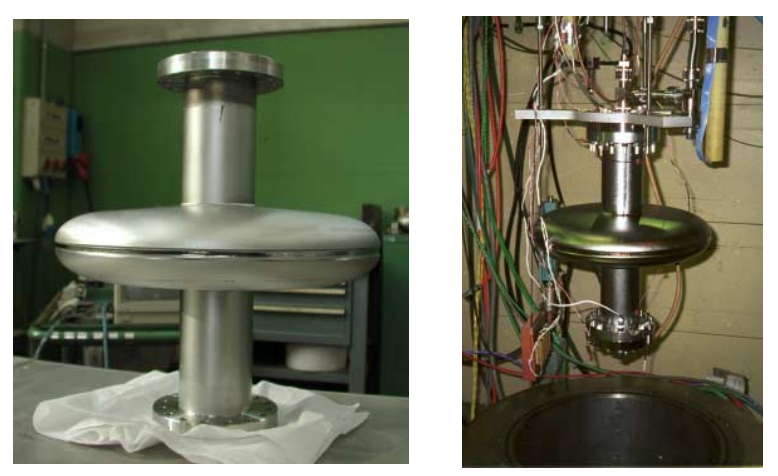

Figure 1. Prototype Z102 after welding in Zanon and Z101 during tests at Saclay

The dies and the cavity half-cells (before electron beam welding) have been measured with a 3D measuring machine at Orsay, in order to verify the fabrication process. The dies were accurate to $1 / 100 \mathrm{~mm}$ precision, and the average cell profiles were found to be $0.2 \mathrm{~mm}$, well within the required tolerances of $0.4 \mathrm{~mm}$.

Two single cell cavities using high purity $(\mathrm{RRR}>250)$ niobium sheets have been fabricated and will be tested as soon as possible.

\subsection{RF measurement infrastructure in LASA}

For the tests of the TRASCO cavities a RF test bench is being set up in our laboratory. The test area consists in a vertical cryostat for RF measurements, a class-10 clean room for the assembly of cavity components and a highpressure water rinse (HPR) system using ultra pure water $(18 \mathrm{M} \Omega \mathrm{cm})$. 
The RF test stand is equipped with a $500 \mathrm{~W}$ amplifier in the UHF band $(400-800 \mathrm{MHz})$ and a rotating fixture in the cryostat allows the thermal mapping of the cavity during the measurements.

The class-10 clean room ( $3 \mathrm{~m}$ times $3 \mathrm{~m}$ ) has been recently completed and put into operation.

The RF test system (amplifier, phase-locked loop and read-out electronics) has been successfully operated last year during the measurements with an existing $500 \mathrm{MHz}$ $\mathrm{SC}$ electron cavity and is currently being integrated in the computerized control and data acquisition system of the facility. A new adjustable insert for the existing large vertical cryostat $(700 \mathrm{~mm}$ diameter, originally designed for another INFN program with $500 \mathrm{MHz}$ SC cavities) has been fabricated, for the measurements of the different TRASCO cavity prototypes (single-cell and multi-cell cavities with different lengths). The insert fits into a smaller (580 $\mathrm{mm}$ diameter) helium tank that allows reducing the helium consumption for the $700 \mathrm{MHz}$ measurements.

The HPR system is being fabricated and will be assembled in the clean room. The ultra pure water system is in operation (providing a $10 \mathrm{l} / \mathrm{min}$ flow of $18 \mathrm{M} \Omega \mathrm{cm}$ water and a stock of 60001 of $1 \mathrm{M} \Omega \mathrm{cm}$ water) and the test area will be ready to receive the first $700 \mathrm{MHz}$ cavities in Fall 2001. A thorough cryogenic test on the vertical cryostat (without any cavity in the insert) has been performed, reaching a temperature of $1.8 \mathrm{~K}$ by pumping on the He bath. The test was particularly aimed at testing the new adjustable cryostat insert and at verifying the natural convection pre-cooling procedure that will be employed during the cooldown, to minimize $\mathrm{He}$ use and to prevent gas mixture between $\mathrm{He}$ and $\mathrm{N}_{2}$ [7].

Finally, an arrangement with a local chemical company is being set up for the buffered chemical polishing (BCP) treatment of the cavities needed before the RF tests.

\section{$2.5 Z 101$ measurements at Saclay}

The first prototype single cell cavity built by Zanon was delivered well before completion of the RF test infrastructure in LASA, and was sent to CEA/Saclay for chemical treatments and RF tests.

The geometrical and electromagnetic parameters of the single cell Z101 cavity are resumed in Table 1.

Table 1. Z101 Cavity parameters

\begin{tabular}{|l|l|}
\hline Geometrical $\beta$ & 0.47 \\
\hline Nominal Frequency [MHz] & 699.6 \\
\hline Ep/Eacc & 2.81 \\
\hline Bp/Eacc [mT/(MV/m)] & 5.39 \\
\hline Geometrical factor [Ohm] & 147.8 \\
\hline Iris bore radius [mm] & 40.0 \\
\hline Cell length (iris to iris) [mm] & 100.0 \\
\hline Wall inclination [deg] & 5.5 \\
\hline Equator ellipse aspect ratio & 1.6 \\
\hline Iris ellipse aspect ratio & 1.3 \\
\hline Cavity Diameter [mm] & 187.04 \\
\hline Length of each beam tube [mm] & 160.0 \\
\hline
\end{tabular}

The cavity was measured in July 2000 and, in spite of the use of reactor grade niobium sheets (niobium specifications were for $\mathrm{RRR}>30$ ), it reached performances in excess of the TRASCO specifications. The Q vs. $E_{a c c}$ curve obtained from the measurements is shown in Figure 2. No evidence of multipacting barriers came from the RF tests. At the accelerating field of approximately $10.5 \mathrm{MV} / \mathrm{m}$ the cavity quenched abruptly.

A BCP with an acid mixture of $\mathrm{HF}, \mathrm{HNO}_{3}$ and $\mathrm{H}_{3} \mathrm{PO}_{4}$ (in the ratio $1: 1: 2$ ) for 115 minutes removed approximately $100 \mu \mathrm{m}$ on both the internal and external surfaces. The treatment ended with HPR with $18 \mathrm{M} \Omega \mathrm{cm}$ ultra pure water and drying in the clean room.

The cavity was then prepared again at Saclay with a different assembly to fit our vertical insert and the cavity will soon be tested again in LASA for the final commissioning of the test bench.

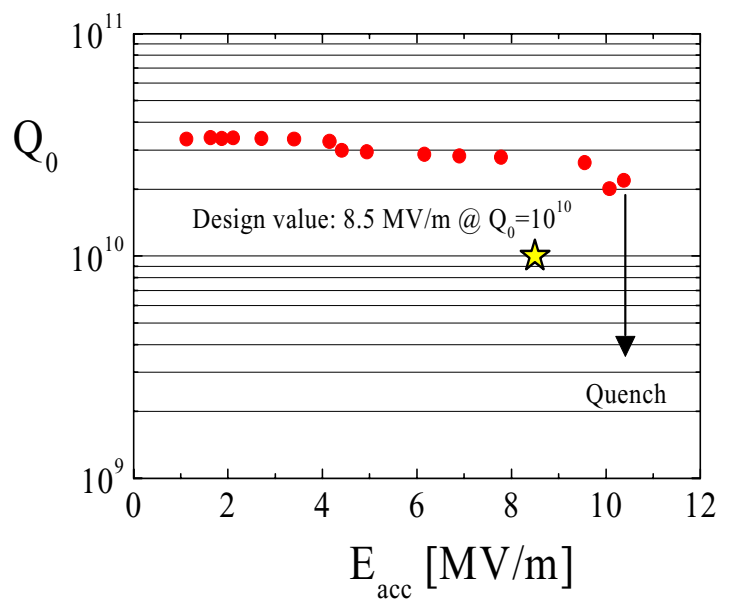

Figure 2: The Q vs. $E_{\text {acc }}$ curve of the Z101 cavity built with reactor grade niobium and tested at Saclay.

From the RF measurements the total surface resistance Rs (at the moderate accelerating field of $1.7 \mathrm{MV} / \mathrm{m}$ ) was evaluated as a function of the temperature, as shown in Figure 3.

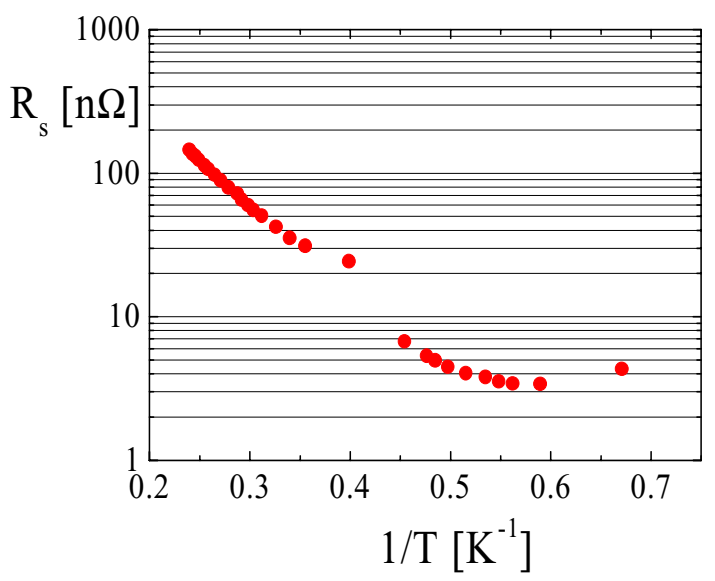

Figure 3: The surface resistance evaluated from the RF measurements. 
The operating $\mathrm{R}_{\mathrm{s}}$ corresponding to the measurements of Figure $2(\mathrm{~T}=1.5 \mathrm{~K})$ is approximately $4 \mathrm{n} \Omega$.

As it can be seen from Figure 3, the low temperature behavior of the surface resistance needs to be investigated with further details, possibly including error bars for the measurement, in order to resolve the inconsistency of an increase of $\mathrm{R}_{\mathrm{s}}$ in the rightmost point, at $1.5 \mathrm{~K}$.

However, the results obtained with this cavity are consistent, in terms of residual resistances, both with the TESLA experience [8] and with the recent measurements of the RIA $\beta=0.5$ single-cell cavities performed at TJNAF (and presented elsewhere in these Proceedings [9]). In all cases the residual surface resistance contributes with a few $n \Omega$ to the total surface resistance.

In both the low- $\beta$ cases (TRASCO and RIA) the surface resistance has been estimated to a few (4-5) $\mathrm{n} \Omega$, and, being the geometrical factors of the cavities very similar, the $Q$ vs. $E_{\text {acc }}$ curves overlap up to the quench field of the TRASCO cavity. Of course, for the $2 \mathrm{~K}$ RIA measurements the dominant contribution to $\mathrm{R}_{\mathrm{S}}$ is the BCS term [10], whereas a $4 \mathrm{n} \Omega$ residual resistance value limited our tests, being the BCS contribution smaller than $1 \mathrm{n} \Omega$. It is worthwhile to note here that our residual resistance value is consistent with that routinely obtained for the TTF cavities [8], while the value obtained at TJNAF for the RIA cavities [9] is surely outstanding. The Z101 TRASCO cavity quench at fields higher than $10.5 \mathrm{MV} / \mathrm{m}$ (corresponding to a magnetic field of nearly $60 \mathrm{mT}$ on the cavity surface) is probably due either to the low thermal conductivity of the low RRR material or to the presence of a small local defect. We also note, that the quench occurs with no apparent $\mathrm{Q}$ degradation and with negligible X-ray emission.

\section{FUTURE TRASCO ACTIVITIES}

The next step of the TRASCO program for the SC cavity R\&D will be the RF tests in Milano of all the 4 single-cell cavities that have been fabricated so far by Zanon.

In particular, the tests with the high RRR niobium single-cell cavities will be important to confirm that high field values can be reached at these low- $\beta$ values, as in the case of the RIA prototype cavities.

Then we will proceed to the fabrication of 2 full 5-cell $\beta=0.5$ cavities, including a fundamental and HOM coupler ports. The possibility to build a 2 or 3 cell cavity for the test of stiffening schemes before building the 5cells is still under investigation.

\section{ACKNOWLEDGEMENTS}

We are greatly indebted with the CEA/Saclay and IN2P3/Orsay SC RF cavities group for the chemical treatments and the tests of the Z101 cavity.

We would also like to thank the support from Zanon, in particular G. Corniani, G. Basoni and M. Festa, for the cavity fabrication, tooling and electron beam welding.

\section{REFERENCES}

[1] The TRASCO_AC Group, "Status Of The High Current Proton Accelerator For The TRASCO Program", Internal Report INFN/TC-00/23, 21 December 2000; http://www.lnf.infn.it/sis.

[2] D. Barni, A. Bosotti, G. Ciovati, C. Pagani, P. Pierini, "SC Cavity Design For The $700 \mathrm{MHz}$ TRASCO Linac", in Proceedings of the 2000 European Particle Accelerator Conference, Vienna, Austria, p. 2019.

[3] J.L. Biarrotte, H. Safa, J.P. Charrier, S. Jaidane, H. Gassot, T. Junquera, J. Lesrel, G. Ciovati, P. Pierini, D. Barni, C. Pagani, "704 MHz Superconducting Cavities For A High Intensity Proton Accelerator", in Proceedings of the Ninth Workshop on RF Superconductivity, Santa Fe, NM USA, 1999.

[4] H. Safa, "Superconducting Proton Linac for Waste Transmutation", in Proceedings of the Ninth Workshop on RF Superconductivity, Santa Fe, NM USA, 1999.

[5] P. Pierini, D. Barni, A. Bosotti, G. Ciovati, C. Pagani, "Cavity Design Tools And Applications To The TRASCO Project", in Proceedings of the Ninth Workshop on RF Superconductivity, Santa Fe, NM USA, 1999.

[6] See http://www.zanon.com for more information.

[7] D. Barni, C. Gesmundo, C. Pagani, G. Varisco, "Beta Tunable $700 \mathrm{MHz}$ Insert With Natural Convection Precooling", in Proceedings of the Ninth Workshop on RF Superconductivity, Santa Fe, NM USA, 1999.

[8] B. Aune et al., "Superconducting TESLA Cavities", Phys. Rev. ST-AB, Vol. 3, 092001 (2000).

[9] W. Hartung et al, "Niobium Cavity Development for the High-Energy Linac of the Rare Isotope Accelerator", in these Proceedings.

[10] See "RF Superconductivity for Accelerators", by H. Padamsee, J. Knobloch, T. Hays, John Wiley, 1998. 